\title{
7 EFEITO DE UM PROGRAMA DE ATIVIDADE FÍSICA NA CAPACIDADE FUNCIONAL PARA O EXERCÍCIO E NÍVEL DE ATIVIDADE FÍSICA DE PESSOAS COM ESQUIZOFRENIA - UM ESTUDO PILOTO
}

\author{
| Eluana Gomes; Tânia Bastos²; Raquel Costa ${ }^{3}$; Rui Corredeira ${ }^{4}$
}

\section{RESUMO}

CONTEXTO: A esquizofrenia é uma doença grave e debilitante. Somado a isso, a maioria das pessoas com esquizofrenia apresenta um estilo de vida pouco saudável, que inclui níveis insuficientes de atividade física diária.

OBJETIVO: O propósito do presente estudo foi avaliar a eficácia de um programa de Atividade Física (AF) de 24 semanas na capacidade funcional para o exercício e no nível de AF de pessoas com esquizofrenia.

MÉTODOS: Nove pessoas (cinco homens e quatro mulheres) com diagnóstico de esquizofrenia participaram durante 24 semanas num programa de AF. O programa contemplou duas sessões por semana, com a duração de 60 minutos/sessão. As avaliações ocorreram no início (M0), após 12 semanas (M1) e 24 semanas (M2). A capacidade funcional e o nível de AF foram avaliados através do Teste de caminhada de 6 minutos e do Questionário Internacional de AF, versão curta, respetivamente.

RESULTADOS: Verificaram-se melhorias significativas na capacidade funcional para o exercício. Apesar da ausência de significância estatística, observou-se um aumento no nível de AF e redução nos valores da circunferência da cintura e da anca entre M0 e M2.

CONCLUSÕES: Os resultados sugerem que o programa de AF pode promover um estilo de vida saudável nas pessoas com esquizofrenia.

\section{PALAVRAS-CHAVE: Espectro da esquizofrenia e outros transtornos psicóticos; Atividade motora; Caminhada}

\section{RESUMEN}

"Efecto de un programa de actividad física en la capacidad funcional para el ejercicio y la actividad física de las personas con esquizofrenia - un estudio piloto"

CONTEXTO: La esquizofrenia es una enfermedad grave y debilitante. Sumado a ello, gran parte de la población presenta un estilo de vida poco saludable, que incluye niveles insuficientes de actividad física diaria.

OBJETIVO: El objetivo del presente estudio fue evaluar la eficacia de un programa de actividad física (AF) de 24 semanas en la capacidad funcional para el ejercicio y en el nivel de AF de personas con esquizofrenia.

MÉTODOS: Nueve personas (cinco hombres e cuatro mujeres) con diagnóstico de esquizofrenia participaron durante 24 semanas en un programa de AF. El programa contempló dos sesiones por semana, con una duración de 60 minutos / sesión. Las evaluaciones ocurrieron al principio (M0), después de 12 semanas (M1) y 24 semanas (M2). La capacidad funcional y el nivel de AF fueron evaluados a través del prueba de caminata de 6 minutos y el Cuestionario Internacional de la Actividad Física - versión corta, respectivamente.

RESULTADOS: Se observaron mejoras significativas en la capacidad funcional para el ejercicio. Aunque no se observaron diferencias estadísticamente significativas, se observó un aumento en el nivel de AF y reducción en los valores de la circunferencia de la cintura y de la cadera entre M0 y M2.

CONCLUSIONES: Los resultados sugieren que el programa de AF puede promover un estilo de vida saludable en las personas con esquizofrenia.

\section{DESCRIPTORES: Espectro de esquizofrenia y otros trastornos} psicóticos; Actividad motora; Caminata

\begin{abstract}
"Effect of a 24-week physical activity program in functional exercise capacity and level of physical activity in people with schizophrenia - a pilot study"

BACKGROUND: Schizophrenia is a most debilitating disease. In addition, much of this people had an unhealthy lifestyle, which includes insufficient daily physical activity levels.

AIM: The purpose of this study was to evaluate the effects of a 24-week physical activity (PA) program in functional exercise capacity and level of PA in people with schizophrenia.

METHODS: Nine persons (five men and four women) with diagnose of schizophrenia participated for 24 weeks in a PA program. The program occurred twice a week, with 60 minutes per session. The evaluations occurred at baseline (M0), after 12 weeks (M1) and 24 weeks (M2). The functional capacity and the level of PA were evaluated through the 6-minute Walk Test and the International Physical Activity Questionnaire - Short Form, respectively.

RESULTS: There were significant improvements in functional exercise capacity. Although there were no significant statistically differences, there was an increase in the level of PA and a reduction in waist and hip circumferences between M0 and M2.

CONCLUSIONS: The results suggest that the PA program can promote a healthy lifestyle in people with schizophrenia.
\end{abstract}

\section{KEYWORDS: Schizophrenia spectrum and other psychotic disorders; Motor activity; Walking}

Submetido em 20-03-2017

Aceite em 11-11-2017

1 Universidade do Porto, Faculdade de Desporto, Centro de Investigação em Atividade Física, Saúde e Lazer, 4200-450 Porto, Portugal, aeluana@yahoo.com.br

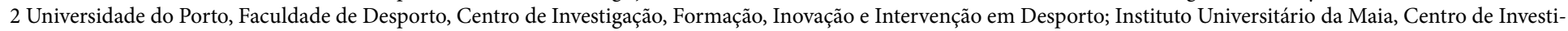
gação em Desporto, Saúde e Desenvolvimento Humano, 4475-690 Maia, Portugal.

3 Universidade do Porto, Faculdade de Desporto, Centro de Investigação em Atividade Física, Saúde e Lazer, 4200-450 Porto, Portugal.

4 Universidade do Porto, Faculdade de Desporto, Centro de Investigação em Atividade Física, Saúde e Lazer, 4200-450 Porto, Portugal

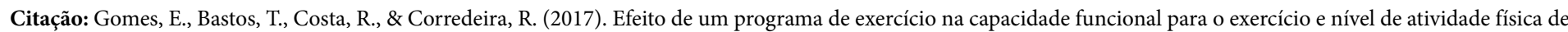
pessoas com esquizofrenia - um estudo piloto. Revista Portuguesa de Enfermagem de Saúde Mental (18), 44-50. doi: 10.19131/rpesm.0191 


\section{INTRODUÇÃO}

As pessoas com esquizofrenia apresentam uma esperança média de vida inferior, bem como um risco de mortalidade duas a três vezes superior à população em geral (Ministério da Saúde, 2008). Em causa estão os inadequados estilos de vida que são comumente reportados pelas pessoas com esquizofrenia. Mais especificamente, a maioria das pessoas com esquizofrenia são sedentárias, ingerem frequentemente bebidas alcoólicas, são fumadores e apresentam uma inadequada dieta alimentar (Faulkner \& Biddle, 1999) Somado a isso, os medicamentos antipsicóticos regularmente utilizados para controlo dos sintomas da doença, podem provocar ganho de peso corporal, bem como o desenvolvimento de doenças cardiovasculares, Diabetes Mellitus e síndrome metabólica (Allison et al., 2009; Attux et al., 2011; Ministério da Saúde, 2008).

Neste sentido, as intervenções psicossociais apresentam-se como essenciais no tratamento da esquizofrenia (Attux et al., 2011). Diferentes modelos de intervenção psicossocial têm sido desenvolvidos nos últimos anos com o objetivo de auxiliar na promoção um estilo de vida saudável nestas pessoas (Allison et al., 2009; Attux et al., 2011; Beebe et al., 2005). Especificamente, os benefícios da prática de atividade física (AF) têm despertado o interesse dos investigadores na área da saúde mental (Faulkner \& Biddle, 1999; Faulkner, Cohn, \& Remington, 2006). Diversos estudos têm demonstrado que a prática de AF regular contribui para a redução do peso corporal (Vancampfort et al., 2011a), da circunferência da cintura (Attux et al., 2011), da percentagem de massa gorda (Beebe et al., 2005), contribuindo ainda para a melhoria da autoestima e de funções cognitivas e sociais (Marzolini, Jensen, \& Melville, 2009; Vaz-Serra et al., 2010). Somado a isso, a prática de AF auxilia na redução dos sintomas negativos da doença (e.g., avolição, dificuldade no discurso, dificuldade nas relações sociais) e na dosagem dos medicamentos antipsicóticos (Vancampfort et al., 2011a). Os sintomas negativos têm um impacto direto na redução da capacidade funcional e, consequentemente, na redução do desempenho físico, social e cognitivo (Vaz-Serra et al., 2010), comprometendo desta forma a realização das atividades da vida diária.

Nesta medida, a prática regular de AF pode contribuir para a redução de custos na saúde pública, uma vez que existe uma relação direta entre a redução de um estilo de vida sedentário e a redução dos cuidados básicos de saúde (Allison et al., 2009; Attux et al., 2011).
Assim, torna-se fundamental promover hábitos regulares de prática de AF nas pessoas com esquizofrenia, bem como desenvolver programas de AF ajustados às suas necessidades (Vancampfort et al., 2012). Importa realçar que, parte dos estudos relacionados com a implementação de programas de AF em pessoas com esquizofrenia incluem pacientes residentes na comunidade, ou seja, indivíduos que não se encontram institucionalizados. Este enfoque é fundamental no processo de reabilitação destes pacientes, uma vez que auxilia na diminuição do número de reinternamentos nos serviços de saúde (Marzolini et al., 2009). Neste contexto, profissionais de saúde mental devem considerar o desenvolvimento de estratégias de adesão destes doentes aos programas de AF (Vancampfort et al., 2011a).

No que diz respeito ao contexto nacional, importa referir que, foi possível identificar apenas um estudo relacionado com a aplicação de um programa de AF para pessoas com esquizofrenia em Portugal (Gomes et al., 2014). O mesmo ofereceu um programa de AF com duração de 16 semanas. A análise do nível de AF foi realizada através da utilização de aparelho acelerómetro. No entanto, é necessário ter em consideração a utilização de instrumentos que sejam de baixo custo e fácil aplicação em contexto clínico (Vancampfort et al., 2011b). A forma mais comum de avaliação dos programas de AF é através de questionários, uma vez que os mesmos são ferramentas para avaliar padrões, frequência, tipo e contexto da AF (Vanheesa et al., 2005).

O Plano Nacional de Saúde Mental 2008-2016 (PNSM) aponta a inexistência de programas de promoção da saúde para a população com doença mental em Portugal. Deste modo, torna-se necessário o desenvolvimento de programas que promovam a melhoria física e, consequentemente, a autonomia destas pessoas na respetiva comunidade, bem como aumentar a produção científica na área da saúde mental (Ministério da Saúde, 2008). O presente estudo piloto pretendeu avaliar a eficácia de um programa de AF de 24 semanas na capacidade funcional para o exercício físico e no nível de AF de pessoas com esquizofrenia.

\section{MÉTODOS}

O presente estudo foi realizado através de uma abordagem longitudinal, no qual se recolheu medidas antropométricas, aplicou-se um teste de terreno e um questionário, antes e após a aplicação de um programa de intervenção de AF, adaptado às caraterísticas dos participantes com diagnóstico de esquizofrenia. 
De forma mais específica, a metodologia utilizada é caraterizada por:

\section{Participantes}

Participaram neste estudo 9 pessoas (homens $=5$; mulheres $=4)$ com diagnóstico de Esquizofrenia e Transtorno Esquizoafetivo, de acordo com o Diagnostic and Statistical Manual of Mental Disorders (DSM-IV-TR; American Psychiatric Association [APA], 2000), residentes na comunidade, com idades entre 27 e 55 anos $(\mathrm{M}=43.11 \pm \mathrm{DP}=9.6)$. Os participantes foram selecionados no serviço de psiquiatria de um Hospital Público da cidade do Porto/Portugal. Após esclarecimento acerca dos objetivos do estudo e a garantia de confidencialidade dos dados, os participantes assinaram o consentimento livre e esclarecidos de acordo com os princípios da Declaração de Helsinki. O estudo foi aprovado pelo Comité de Ética do Hospital de proveniência da amostra ( $\left.\mathrm{n}^{\circ} 21529\right)$.

A Tabela 1 apresenta a caracterização da amostra relativamente ao grau de escolaridade, estado civil e condição de residência dos participantes.

Tabela 1 - Caracterização dos Participantes Quanto ao Grau de Escolaridade, Estado Civil e Condição de Residência ( $\mathrm{N}=9$ )

\begin{tabular}{|l|l|}
\hline Características & $\mathbf{n}$ \\
\hline Grau de escolaridade & 6 \\
\hline Ensino básico & 2 \\
\hline Ensino médio & 1 \\
\hline Ensino superior & 7 \\
\hline Estado Civil & 2 \\
\hline Solteiro & \\
\hline Casado & 5 \\
\hline Vive com & 2 \\
\hline Mãe/Pai & 1 \\
\hline Esposa/Esposo & 1 \\
\hline Sozinho & \\
\hline Avós & \\
\hline
\end{tabular}

No que se refere à caracterização do regime de tratamento medicamentoso, todos os participantes utilizavam antipsicóticos atípicos, bem como ansiolíticos ( $\mathrm{n}$ $=4)$ e antidepressivos $(n=2)$. Todos os participantes indicaram que não praticavam AF regular há pelo menos 2 anos. No que diz respeito às vivências desportivas anteriores, os participantes indicaram as aulas de educação física durante o percurso escolar, assim como atividades de musculação, culturismo, ténis, futebol e spinning.
Caracterização do Programa de Atividade Física Os participantes realizaram durante 24 semanas um programa de AF com sessões bissemanais e duração aproximada de 60 minutos/sessão. As sessões de AF apresentavam a seguinte estrutura: exercícios de alongamento dos grandes grupos musculares (aproximadamente 10 minutos), abdominal, exercícios reduzidos da modalidade futebol e voleibol (aproximadamente 40 minutos) e exercícios de retorno à calma (aproximadamente 5 a 10 minutos).

Durante as sessões, a intensidade dos exercícios foram controladas através da Escala de Borg (Borg, 1998). Foi utilizado um diário de campo para registo da presença dos participantes às sessões e observações sobre a aula (participação nas atividades, reações sobre as atividades propostas). A aplicação da bateria de testes ocorreu no início (M0), após 12 semanas (M1) e no final de 24 semanas (M2) de aplicação do programa de AF.

\section{Instrumentos}

De forma a responder aos objetivos propostos, uma bateria de testes foi aplicada em três momentos de avaliação (M0; M1 e M2). A bateria de testes é composta por diferentes tipos de instrumentos (i.e., medições, questionário e teste de terreno), que se encontram validados e são frequentemente aplicados em pessoas com esquizofrenia. De uma forma mais específica, os instrumentos utilizados no presente estudo foram:

\section{Medidas Antropométricas}

Foram avaliadas as medidas antropométricas relativas à altura e peso corporal, para posterior cálculo do índice de massa corporal (IMC). A altura foi medida com um estadiómetro da marca Holtain (Holtain Ltd., Crymmych, UK) e o peso corporal foi medido com uma balança eletrónica Welmy. Procedeu-se ao cálculo do IMC através da fórmula $\left(\mathrm{kg} / \mathrm{m}^{2}\right)$. A circunferência da cintura e da anca foram medidas com uma fita métrica e registadas em centímetros.

\section{Nível de Atividade Física}

O Questionário Internacional de Atividade Física, versão curta (IPAQ-SF) (Craig et al., 2003) foi utilizado para avaliar o nível de AF dos participantes. Este questionário fornece informações sobre o número de dias e minutos de caminhada e de atividades de intensidade moderada e vigorosa ao longo de uma semana. O IPAQSF é considerado um instrumento confiável para avaliar o nível de AF em pessoas com esquizofrenia (Faulkner et al., 2006). 


\section{Capacidade Funcional para o Exercício}

O Teste de Caminhada de 6 minutos (TC6) (American Thoracic Society, 2002) avalia a capacidade funcional para o exercício. É um teste de esforço submáximo e avalia, de forma global e integrada, as respostas do sistema cardiovascular e pulmonar (American Thoracic Society, 2002).

As vantagens descritas na literatura sobre a aplicação do TC6 são: i) correlação com VO2 (i.e., consumo máximo de oxigénio); ii) fácil aplicação por parte dos investigadores; iii) baixo custo; iv) relação com a execução das atividades de vida diária. O TC6 é amplamente utilizado em estudos com pessoas com esquizofrenia (Beebe et al., 2005; Marzolini et al., 2009; Vittaca, Paneroni, Comini, \& Bianchi, 2013). A validação preliminar do TC6 para este grupo foi desenvolvido em nível internacional (Vancampfort et al., 2011b) e nacional (Gomes et al., 2016).

\section{Análise Estatística}

Procedeu-se à análise dos dados através da utilização do Software Statistical Package for the Social Sciences (SPSS), versão 21.0.

Foram utilizadas as medidas descritivas convencionais. Foi utilizado o Teste de Friedman para explorar possíveis diferenças nas variáveis contínuas nos três momentos de avaliação (M0, M1 e M2) no programa estruturado de AF. O nível de significância foi estabelecido em $\mathrm{p}<0.05$.

\section{RESULTADOS}

A média de assiduidade dos participantes nas sessões foi de $85 \%$ e a adesão ao programa de AF foi de $90 \%$ ao longo de 24 semanas. A estatística descritiva relativa aos dados antropométricos, TC6 e IPAQ-SF nos três momentos de avaliação do programa de AF encontrase apresentada na Tabela 2.

Tabela 2 -Valores Médios e Desvios Padrão para as Variáveis Antropométricas, TC6 e IPAQ-SF no Início (M0), Após 12 Semanas (M1) e no Fim de 24 Semanas de Intervenção (M2)

\begin{tabular}{|l|c|c|c|c|}
\hline & M0 & M1 & M2 \\
\hline Peso Corporal (Kg) & $77.73 \pm 16.14$ & $77.73 \pm 15.37$ & $78.03 \pm 15.05$ & 0.787 \\
\hline IMC $\left(\mathbf{K g} / \mathbf{m}^{2}\right)$ & $28.09 \pm 4.80$ & $28.13 \pm 4.60$ & $28.26 \pm 4.51$ & 0.794 \\
\hline Circunferência cintura (cm) & $97.11 \pm 13.68$ & $95.11 \pm 15.62$ & $91.66 \pm 19.61$ & 0.692 \\
\hline Circunferência anca (cm) & $104.00 \pm 9.39$ & $100.55 \pm 12.06$ & $96.27 \pm 19.83$ & 0.193 \\
\hline TC6 (m) & $464.77 \pm 79.50$ & $552.38 \pm 103.86$ & $587.55 \pm 00.06 a, b$ & $<0.001$ \\
\hline IPAQ-SF & \multicolumn{3}{|c|}{} \\
\hline Total AF (min.) & $158.33 \pm 94.60$ & $316.66 \pm 281.08$ & $235.00 \pm 75.69$ & 0.224 \\
\hline AF vigorosa (min.) & $17.22 \pm 28.84$ & $2.77 \pm 5.65$ & $23.88 \pm 45.94$ & 0.776 \\
\hline AF moderada (min.) & $62.22 \pm 35.00$ & $159.44 \pm 137.94$ & $101.66 \pm 68.64$ & 0.156 \\
\hline AF caminhada (min.) & $78.88 \pm 73.94$ & $154.44 \pm 182.61$ & $109.44 \pm 23.27$ & 0.313 \\
\hline Total AF (MET/min./semana) & $569.83 \pm 95.68$ & $1281.66 \pm 1021.63$ & $958.94 \pm 28.71$ & 0.224 \\
\hline
\end{tabular}

$\mathrm{AF}=$ Atividade Física; $\mathrm{MET}=$ Equivalente Metabólico. $\mathrm{aM} 0<\mathrm{M} 1 ; \mathrm{bM} 0<\mathrm{M} 2$

Figura 1 -TC6 no início (M0), após 12 semanas (M1) e no fim de

Apesar de não se terem verificado diferenças estatisticamente significativas, observou-se entre M0 e M2 uma redução nos valores da circunferência da cintura e da anca.

No que diz respeito à capacidade funcional para o exercício verificaram-se melhorias significativas nos valores médios de distância percorrida durante os três momentos de avaliação do TC6 $(\mathrm{X} 2 \mathrm{~F}(2)=12.400 ; \mathrm{p}<0.001)$. Tal como ilustra a figura 1 , a média de distância percorrida aumentou significativamente entre o M0 e M1 ( $\mathrm{p}=$ $0.01)$ e entre M0 e M2 ( $<$ <.001). 24 semanas de intervenção (M2).

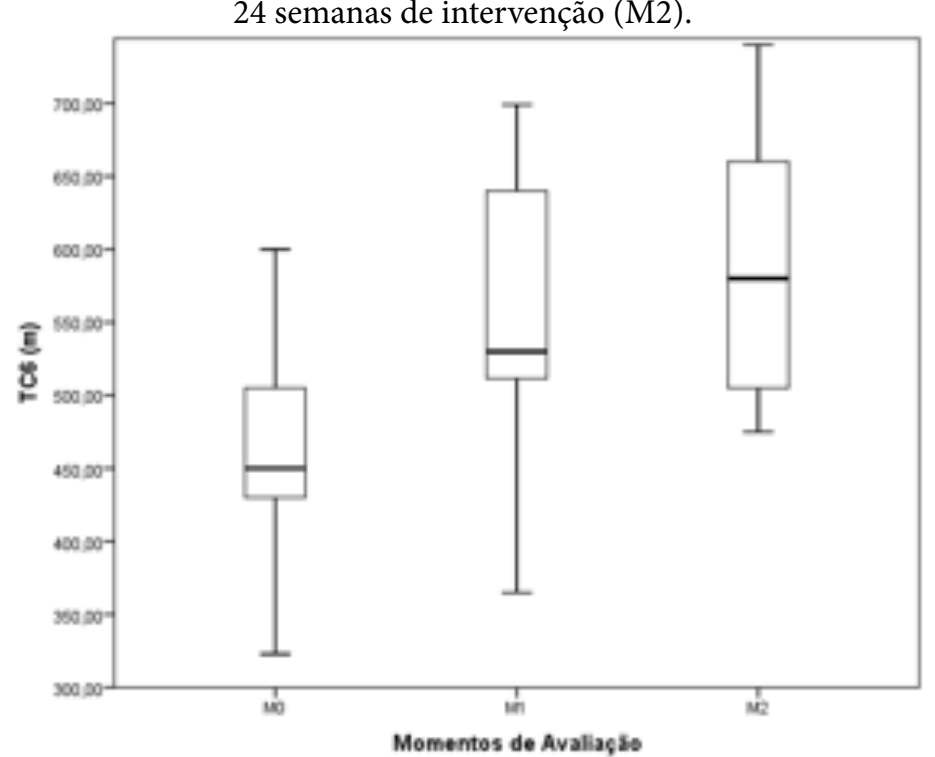


Por último, verificou-se um aumento na média do nível de AF (i.e., total minutos de AF, minutos de AF vigorosa/semana, minutos de AF moderada/semana, minutos de caminhada/semana e total de MET/minuto/semana) no final das 24 semanas de intervenção, ainda que não tenham sido observadas diferenças estatisticamente significativas. No entanto, ainda que a média do nível de AF relatado pelos participantes tenha aumentado de M0 para M2 foi notória uma oscilação dos valores entre os três momentos de avaliação (tabela 2).

\section{DISCUSSÃO}

O presente estudo piloto avaliou a eficácia de um programa de AF de 24 semanas na capacidade funcional para o exercício e no nível de AF de pessoas com esquizofrenia. A média de frequência dos participantes ao programa foi de $67 \%$, corroborando com outros programas de AF relatados na literatura especializada (Marzolini et al., 2009; Vittaca et al., 2013)

O programa de AF indicou a melhoria da capacidade funcional para o exercício dos participantes. Esta evidência assume particular importância uma vez que o aumento da média de distância caminhada favorece a melhoria dos parâmetros fisiológicos (i.e., sistema cardiovascular e respiratório) e das capacidades motoras (i.e., equilíbrio e força) influenciando, deste modo, a capacidade funcional para a realização das atividades da vida diária das pessoas com esquizofrenia (Vancampfort et al., 2011a). Os resultados deste estudo piloto são similares a estudos prévios nos quais foram observadas melhorias significativas na média de distância caminhada após 24 semanas de aplicação de um programa de caminhada e ginásio (bicicleta ergométrica e abdominal) (Vittaca et al., 2013). Em contrapartida, foram encontrados na literatura especializada alguns estudos no qual não foram observadas melhorias significativas na capacidade funcional para o exercício após a implementação de um programa de AF de 12 e 16 semanas, respetivamente (Beebe et al., 2005; Marzolini et al., 2009). Possivelmente, a duração e os exercícios desenvolvidos no programa aplicado no presente estudo promoveu melhorias significativas neste parâmetro.

Relativamente ao nível de $\mathrm{AF}$, não se verificaram alterações significativas ao fim de 24 semanas de aplicação do programa de AF, ainda que se tenham obtido melhorias em todas as variáveis avaliadas pelo IPAQ-SF. A ausência de significância estatística poderá estar relacionada, com o reduzido número de participantes no estudo $(n=9)$.
Neste contexto, sugere-se que estudos futuros aumentem o tamanho da amostra de modo a permitir alcançar resultados mais conclusivos acerca do nível de AF de pessoas com esquizofrenia envolvidos em programas dessa natureza. No entanto, importa realçar a importância do programa no aumento nos níveis de AF dos participantes. Isto porque, o aumento no nível de AF está associado a redução do risco de doenças crónicas (Faulkner \& Biddle, 1999; Vancampfort et al., 2012). Ainda no que diz respeito ao nível de AF, de uma forma geral, foi observada uma redução do M1 para M2. A prática regular de AF, proporcionada pelo programa, possivelmente influenciou a perceção de intensidade (exercício moderado ou vigoroso) dos participantes, uma vez que estas pessoas relataram apresentar estilo de vida sedentário há pelo menos dois anos. Após 12 semanas de intervenção julgamos que a sua perceção inicial de intensidade do exercício alterou-se devido aos efeitos do programa na adaptação fisiológica do organismo à prática regular de AF. No entanto, a não progressão do nível de AF dos participantes ao longo programa (M1-M2) sugere que, possivelmente, houve um desfasamento entre a perceção de intensidade do exercício reportada pelos participantes no M0. Esta situação poderá ser consequência das características associadas à esquizofrenia, nomeadamente as alterações no pensamento e a perceção da realidade (Awad \& Voruganti, 2012). Outra possível explicação para o resultado anteriormente citado poderá estar relacionada com a possível dificuldade dos participantes em classificarem as atividades moderadas e vigorosas durante a aplicação do IPAQ-SF, situação frequentemente descrita na literatura (Faulkner et al., 2006).

Somado a isso, a discussão dos resultados relativos ao nível de AF se encontra limitada uma vez que este é o único estudo ao nível nacional e internacional, que seja do nosso conhecimento, no qual o IPAQ-SF foi utilizado no contexto desportivo em pessoas com esquizofrenia, residentes na comunidade.

Relativamente às medidas antropométricas, observouse uma diminuição da circunferência da cintura e da anca após 24 semanas de intervenção, apesar da ausência de significância estatística. A tendência verificada no presente estudo torna-se relevante para a melhoria da saúde das pessoas com esquizofrenia, uma vez que o aumento da circunferência da cintura e anca estão relacionados com o risco de doenças cardiovasculares e Diabetes Mellitus tipo 2 (The American Journal Of Clinical Nutrition, 1998). 
Não foram verificadas alterações significativas no peso corporal e IMC ao longo do programa de AF. Estes resultados corroboram com estudos sobre programas desta natureza aplicados em pessoas com esquizofrenia (Beebe et al., 2005; Marzolini et al., 2009). A ausência de controlo dos hábitos alimentares dos participantes (Marzolini et al., 2009), bem como a falta de avaliação da massa magra e massa gorda dos participantes ao longo do programa de AF, dificulta a discussão sobre os resultados encontrados. Estudos que implementaram programas de AF com acompanhamento nutricional apresentaram melhorias no peso corporal e IMC (Attux et al., 2011).

\section{CONCLUSÕES}

O presente estudo indicou a melhoria da capacidade funcional para o exercício dos participantes do programa de AF proposto.

Apesar de não se terem verificado diferenças estatisticamente significativas, observou-se um aumento no nível de AF e redução nos valores da circunferência da cintura e da anca entre M0 e M2.

Os profissionais de saúde mental devem também considerar intervenções não farmacológicas que promovam efeitos benéficos na saúde física e mental de cada pessoa com esquizofrenia.

Neste sentido, programas de AF têm sido identificados como uma possível estratégia na promoção de um estilo de vida saudável para estas pessoas. Atualmente, a enfermagem é solicitada para dar sua contribuição numa proposta multidisciplinar, ajudando a pessoa a enfrentar o sofrimento psíquico.

O desafio que se faz presente é o da continuidade do aprofundamento de conhecimento dos benefícios, bem como do aprimoramento de estratégias utilizadas nos programas desta natureza nas pessoas com esquizofrenia.

Neste sentido, futuros estudos devem desenvolver pesquisas de natureza experimental, com seleção aleatória da amostra, incluir novas variáveis para a análise (fatores que influenciam a motivação para o exercício, hábitos alimentares, avaliar se os benefícios são mantidos por um longo período de tempo, entre outros).

Estas ações poderão oferecer ferramentas para o desenvolvimento de um programa mais eficaz na promoção de um estilo de vida saudável para pessoas com esquizofrenia.

\section{REFERÊNCIAS BIBLIOGRÁFICAS}

Allison, D. B., Newcomer, J. W., Dunn, A. L., Blumenthal, J. A., Fabricatore, A. N., Daumit, G. L., \& Alpert, J. E. (2009). Obesity among those with mental disorders: A National Institute of Mental Health Meeting report. American Journal of Preventive Medicine, 36(4), 341350. doi: 10.1016/j.amepre.2008.11.020

American Thoracic Society. (2002). ATS statement: Guidelines for the six-minute walk test. American Journal of Respiratory \& Critical Care Medicine, 166(1), 111-117. doi: 10.1164/rccm.166/1/111

Attux, C., Martini, L. C., de Araújo, C. M., Roma, A. M., Reis, A. F., \& Bressan, R. A. (2011). The effectiveness of a non-pharmacological intervention for weight gain management in severe mental disorders: Results from a national multicentric study. Revista Brasileira de Psiquiatria, 33(2), 117-121.

Awad, A. G., \& Voruganti, L. N. P. (2012). Measuring quality of life in patients with schizophrenia: An update. Pharmacoeconomics, 30(3), 183-195. doi: 10.2165/11594470-000000000-00000

Beebe, L. H., Tian, L., Morris, N., Goodwin, A., Allen, S. S., \& Kuldau, J. (2005). Effects of exercise on mental and physical health parameters of persons with schizophrenia. Issues in Mental Health Nursing, 26(6), 661-676. doi: 10.1080/01612840590959551

Borg, G. (1998). External, physiological and psychological factors and perceived exertion. In G. Borg, Borg's perceived exertion and pain scales (pp. 68-74). Champaign, IL: Human Kinetics.

Craig, C. L., Marshall, A. L., Sjöström, M., Bauman, A. E., Booth, M. L., Ainsworth, B. E., ... \& Sallis, J. F. (2003). International physical activity questionnaire: 12 -country reliability and validity. Medicine \& Science in Sports \& Exercise, 35(8), 1381.

Faulkner, G., \& Biddle, S. (1999). Exercise as an adjunct treatment for schizophrenia: A review of the literature. Journal of Mental Health, 8(5), 441-457. doi: $10.1080 / 09638239917157$ 
Faulkner, G., Cohn, T., \& Remington, G. (2006). Validation of a physical activity assessment tool for individuals with schizophrenia. Schizophrenia research, 82, 225231. doi: $10.1016 /$ j.schres.2005.10.020

Gomes, E., Bastos, T., Probst, M., Ribeiro, J., Silva, G., \& Corredeira, R. (2016). Reliability and validity of 6MWT for outpatients with schizophrenia: A preliminary study. Psychiatry Research, 237, 37-42. doi: 10.1016/j.psychres.2016.01.066

Gomes, E., Bastos, T., Probst, M., Ribeiro, J. C., Silva, G., \& Corredeira, R. (2014). Effects of a group physical activity program on physical fitness and quality of life in individuals with schizophrenia. Mental Health and Physical Activity, 7(3), 155-162. doi: 10.1016/j. mhpa.2014.07.002

Marzolini, S., Jensen, B., \& Melville, P. (2009). Feasibility and effects of a group-based resistance and aerobic exercise program for individuals with severe schizophrenia: A multidisciplinary approach. Mental Health and Physical Activity, 2(1), 29-36. doi: 10.1016/j. mhpa.2008.11.001

Ministério da Saúde. (2008). Plano Nacional de Saúde Mental 2007-2016 - Resumo Executivo. Lisboa: Coordenação Nacional para a Saúde Mental.

The American Journal Of Clinical Nutrition. (1998). Clinical guidelines on the identification, evaluation, and treatment of overweight and obesity in adults: Executive summary. Expert panel on the identification, evaluation, and treatment of overweight in adults. The American Journal Of Clinical Nutrition, 68(4), 899-917.

Vancampfort, D., Knapen, J., Probst, M., Scheewe, T., Remans, S., \& De Hert, M. (2012). A systematic review of correlates of physical activity in patients with schizophrenia. Acta Psychiatrica Scandinavica, 125(5), 352362. doi: 10.1111/j.1600-0447.2011.01814.x

Vancampfort, D., Probst, M., Sweers, K., Maurissen, K., Knapen, J., \& De Hert, M. (2011a). Relationships between obesity, functional exercise capacity, physical activity participation and physical self perception in people with schizophrenia. Acta Psychiatrica Scandinavica, 1-8. doi: 10.1111/j.1600-0447.2010.01666
Vancampfort, D., Probst, M., Sweers, K., Maurissen, K., Knapen, J., \& De Hert, M. (2011b). Reliability, minimal detectable changes, practice effects and correlates of the 6-min walk test in patients with schizophrenia. Psychiatry research, 187(1-2), 62-67. doi: 10.1016/j.psychres.2010.11.027

Vanheesa, L., Lefevreb, J., Philippaerts, R., Martensa, M., Huygens, W., Troostersa, T., \& Beunenb, G. (2005). How to assess physical activity? How to assess physical fitness? European Journal of Cardiovascular Prevention and Rehabilitation, 12, 102-114.

Vaz-Serra, A., Palha, A., Figueira, M. L., Bessa-Peixoto, A., Brissos, S., Casquinha, P., ... \& Teixeira, J. M. (2010). Cognição, cognição social e funcionalidade na esquizofrenia. Acta Medica Portuguesa, 23(6), 1043-1058.

Vittaca, M., Paneroni, M., Comini, L., \& Bianchi, L. (2013). Efficacy of six months physical activity on exercise capacity in patients with mental disorders (resident in a community service). Minerva psychiatrica, 54(1), 71-82. 